\title{
Efecto adyuvante del consumo de nutrientes específicos durante tratamientos antineoplásicos
}

\section{Adjuvant effect of the consumption of specific nutrients during antineoplastic treatments}

\author{
Karla Mariana González Bravo ${ }^{a}$, Esther Ramírez-Moreno ${ }^{b}$, Araceli Polo-Ortíz ${ }^{c}$, Luis \\ Delgado-Olivares ${ }^{d}$
}

\begin{abstract}
:
In Mexico, cancer represents the third cause of mortality, being obesity a trigger for its appearance; while the condition and therapy themselves cause a series of conditions that deteriorate the quality of life of those who suffer it. The relationship between nutritional status and the appearance and treatment of chronic non-communicable diseases has led to search in nutrition for preventive and palliative support. The bibliographic review was carried out in PubMed, Cochrane, Scielo and Google Academic databases; using as keywords: Nutrition, Cancer, Cancer Dietary Treatment, Coadjuvant Therapy, Bioactive Compound and Functional Food. Those articles from 2013 to date were included, excluding those that obtained inconclusive results or were carried out in animals. 21 articles with relevant information on the consumption or supplementation of diverse antioxidants, fat acids and vitamins were included. According to the temporality of the disease, the compounds would have an effect as chemopreventive, chemotherapeutic or palliative. Also being able to act through epigenetic regulation given the exposure of genes to the environment, in this case, food. Although the compounds analysed have a protective, antiinflammatory, antitumor and antiproliferative effect, among other more specific ones, further research is necessary to determine recommended doses and timing of their consumption; in addition to ruling out risks derived from it.
\end{abstract}

\section{Keywords:}

Cancer, nutrition, bioactive compounds, coadjuvant therapy.

\section{Resumen:}

En México el cáncer representa la tercera causa de mortalidad encontrándose la obesidad como un desencadenante para su aparición; mientras que el padecimiento y terapia mismos provocan una serie de afecciones que deterioran la calidad de vida de quien lo padece. La relación entre el estado nutricional y la aparición y tratamiento de enfermedades crónicas no transmisibles ha llevado a buscar en la nutrición un apoyo como preventivo y paliativo. Se realizó la revisión bibliográfica en las bases PubMed, Cochrane, Scielo y Google académico; utilizando como palabras clave: Nutrición, Cáncer, Tratamiento Dietético de Cáncer, Terapia Coadyuvante, Compuesto Bioactivo y Alimento Funcional. Se incluyeron aquellos artículos de 2013 a la fecha; excluyendo los que obtuvieran resultados no concluyentes y fueran realizados sobre animales. Se incluyeron 21 artículos con información relevante sobre el consumo o suplementación de diversos antioxidantes, ácidos grasos y vitaminas. De acuerdo a la temporalidad de la enfermedad, los compuestos tendrían efecto como quimiopreventivos, quimioterapéuticos o paliativos. Pudiendo actuar también, mediante regulación epigenética dada la exposición de los genes al medio, en este caso, los alimentos. Si bien los compuestos analizados tienen un efecto, en general, protector, antiinflamatorio, antitumoral y antiproliferativo, entre otros más específicos; es necesaria una mayor investigación que consiga determinar dosis recomendadas y temporalidad de su consumo, además de descartar riesgos derivados de su consumo.

\section{Palabras Clave:}

\footnotetext{
${ }^{a}$ Universidad Autónoma del Estado de Hidalgo, https://orcid.org/0000-0002-2024-6548, Email: go216981@uaeh.edu.mx

b Universidad Autónoma del Estado de Hidalgo, http://orcid.org/0000-0002-9928-8600, Email: esther_ramirez@uaeh.edu.mx

c Universidad Autónoma del Estado de Hidalgo, https://orcid.org/0000-0001-5561-2221, Email: araceli_ortiz4208@uaeh.edu.mx

d Autor de Correspondencia, Universidad Autónoma del Estado de Hidalgo, https://orcid.org/0000-0002-3506-8393, Email: ldelgado@uaeh.edu.mx
} 


\section{Introducción}

Desde hace aproximadamente dos décadas, el cáncer representa la tercera causa de mortalidad a nivel nacional; sin embargo, establecer una tasa de morbilidad y mortalidad resulta complicado, puesto que se ha centralizado en un mismo diagnóstico un conjunto de enfermedades que actúan de manera distinta y a distintos niveles sistémicos; encontrándose en el año 2015, como las neoplasias más frecuentes: pulmón, próstata, hígado y vías biliares, mama y gástrico ${ }^{1}$. Más del $90 \%$ de las causales se dan por factores ambientales y estilos de vida, principalmente tabaquismo, alcohol, alimentación y obesidad. Mientras que, entre el $5 \%$ y el $10 \%$ se debe a factores genéticos ${ }^{2}$.

En la actualidad, los cambios en el estilo de vida han llevado a la modificación de los patrones de alimentación, donde se ha incrementado la ingesta de alimentos procesados y ultraprocesados, limitado el consumo de alimentos naturales, lo que ha causado un incremento en los índices de sobrepeso y obesidad. Este último, puede ser un desencadenante de cáncer, a través de diversos procesos detonantes como son: hiperinsulinemia, resistencia a la insulina, incremento en la liberación de leptina y factor de necrosis tumoral. Además, se ha demostrado que la malnutrición en cualquiera de sus formas, con un balance energético muy acentuado hacia un alto o reducido consumo de calorías, conlleva a deficiencias metabólicas que alteraran la función del sistema inmune y la acción protectora contra el cáncer ${ }^{3}$. En el caso de las enfermedades neoplásicas, tanto la enfermedad como la terapia, son causales de efectos adversos como anorexia, caquexia, diarrea, vómito, fiebre, neuropatía, cansancio y dolor, entre otros. Por lo que es común la búsqueda de terapias adyuvantes o alternativas, que de manera sinérgica incrementen los beneficios terapéuticos y consigan mitigar los efectos que demeritan la calidad de vida del paciente, las cuales deben ser tomadas con mucho cuidado ${ }^{4}$. El $72.6 \%$ de los pacientes que recurren a estas terapias, utilizan compuestos herbales o vegetales; mientras que el $27.4 \%$ restante, se apoyan de productos procesados o animales ${ }^{5}$.

Se ha estudiado la asociación entre nutrientes específicos y aparición y evolución de enfermedades neoplásicas, encontrando resultados favorables; entre los que destacan la ingesta elevada de fibra, vitaminas y ácidos grasos, que puede tener un efecto protector, antiinflamatorio, antitumoral y antiproliferativo ${ }^{2}$.

Los compuestos bioactivos o nutracéuticos, son componentes de los alimentos que, aunque no son esenciales tienen efectos benéficos a la salud, por lo que son utilizaos en la prevención y tratamiento de diversas enfermedades entre ellas el cáncer. Estos se encuentran en pequeñas cantidades en alimentos de origen vegetal, así como en aquellos ricos en lípidos, ejercen actividades protectoras y moduladoras sobre diversas funciones biológicas, por lo que durante muchos años han sido utilizados como antinflamatorios, antidepresivos, antimicrobianos y como agentes anticancerígenos, esto último mediante la inducción de apoptosis, inhibición de células cancerígenas, supresión de tumores, bloqueo de la invasión y metástasis ${ }^{6}$. Así, por ejemplo, la capsaicina, contenida en el chile ha demostrado que disminuye la proliferación de células cancerígenas. También la fibra, presente en los frijoles, tiene efectos antiproliferativos induciendo la apoptosis de células cancerígenas ${ }^{7}$.

El uso de estos compuestos bioactivos y alimentos funcionales, tiene como finalidad evitar y corregir deficiencias además de ser de ayuda en la tolerancia de tratamientos, reducir efectos secundarios y en general; mejorar la calidad de vida ${ }^{3}$. Sin embargo, muchos de estos carecen de una evidencia bien controlada y fundamentada; encontrándose incluso, efectos negativos y hasta tóxicos posteriores a su consumo ${ }^{5}$. Por lo que el objetivo de esta revisión es recopilar información referente a nutrientes específicos y su relación con la aparición y desarrollo de enfermedades neoplásicas, así como los beneficios derivados de su ingesta; promoviendo su uso como terapia coadyuvante para enfermedades neoplásicas.

\section{Materiales y métodos}

Se realizó una búsqueda de estudios publicados en las bases diferentes bases de datos utilizando como palabras clave Nutrición, Cáncer, Tratamiento Dietético de Cáncer, Terapia Coadyuvante, Compuesto Bioactivo y Alimento Funcional. Se revisaron 36 artículos de 2013 a la fecha. Sin embargo, solo se seleccionaron 21 artículos; 9 de PubMed, 6 de Scielo, 5 de Google académico, y 1 de Cochrane, que incluyen información relevante de referente a compuestos bioactivos especíicos (antioxidantes, polifenoles, vitaminas E y D, fibra, EPA/DHA, isotiocianatos, glutamina, lectinas, probióticos y prebióticos) utilizados como terapia alternativa durante enfermedades neoplásicas.

\section{Resultados}

La etiopatogenia del cáncer está dada por factores modificables, dónde se encuentra la nutrición; y no 
modificables; principalmente los factores genéticos, en los que, sin embargo, se ha podido demostrar una influencia dada por la alimentación ${ }^{3}$. Al demostrarse una asociación entre el consumo de alimentos y la incidencia de enfermedades crónicas no transmisibles, es necesario considerar la utilidad de una adecuada nutrición en la prevención de tales enfermedades, y posteriormente, durante su tratamiento, para atenuar y controlar comorbilidades asociadas ${ }^{8}$.

Un estilo de vida saludable, alimentación correcta, actividad física y un índice de masa corporal adecuado, contribuyen en la disminución de dicha ocurrencia en un 30 a 40\%; por el contrario, una alimentación basada en productos procesados aumenta hasta el 40\% la posibilidad de modificar las células neoplásicas ${ }^{9}$.

Durante el tratamiento del cáncer, sea este quimioterapia, radioterapia o cirugía y desde la aparición de la enfermedad; las afecciones van más allá del órgano blanco; disminuyendo directamente la calidad de vida del paciente. Se puede esperar fatiga, dolor, depresión, y en el tracto gastrointestinal, diarrea, incontinencia fecal, incremento en la frecuencia de defecación, sangrado rectal y exceso de flatulencia ${ }^{10}$, que derivan a su vez, en pérdida de peso, masa muscular y masa grasa; provocando así una serie de enfermedades secundarias que podrían complicar aún más el estado de salud del paciente y su correcta recuperación.

Tabla 1. Clasificación de suplementos en cuanto a su temporalidad y efecto

\begin{tabular}{lll}
\hline Clasificación & Suplemento & Efecto \\
\hline Quimiopreventivos & Retinoides, índoles y ácido fólico & $\begin{array}{l}\text { En el desarrollo, crecimiento celular y apoptosis. } \\
\text { Retardo en el desarrollo de cáncer. }\end{array}$ \\
Quimioterapéuticos & $\begin{array}{l}\text { Vitamina E } \\
\text { Vitamina E, probióticos, selenio. }\end{array}$ & $\begin{array}{l}\text { Control de peroxidación y reducción de estrés oxidativo. } \\
\text { Reducción de efectos neurotóxicos. } \\
\text { Paliativos }\end{array}$ \\
& & Repoblación bacteriana en el intestino delgado.
\end{tabular}

Fuente: (Arrellano et al., 2013)

La búsqueda de alternativas dietéticas que ayuden en la disminución de la sintomatología asociada al tratamiento, así como una mejora en el mismo e inclusive la prevención de enfermedades secundarias o futuras resulta imperante, y una de las principales opciones actualmente se encuentra en los suplementos dietéticos o alimentos funcionales. Sin embargo, existe una clasificación que determina en qué estadio del padecimiento se debería suplementar (tabla 1) ${ }^{10}$ :

a) Quimiopreventivos: Estos se administran durante las primeras etapas neoplásicas e inclusive, previenen la aparición de cáncer. Principalmente se han estudiado retinoides, indoles y ácido fólico. Los primeros, derivados de vitamina $A$, actúan en forma de ácido retinoico con una acción directa sobre receptores nucleares. En el caso de los indoles, entre los que destaca el indol-3-carbinol que ha sido asociado a un efecto antiproliferativo de células cancerosas ${ }^{10}$.

b) Quimioterapéuticos: Tienen un efecto anticancerígeno y actúan de manera directa o indirecta. La peroxidación lipídica de ácidos grasos poliinsaturados (AGPI) es un efecto del estrés oxidativo, que de no controlarse deriva en la generación de radicales libres y a su vez, en la inducción de carcinogénesis. En este caso el $\alpha$ tocoferol, reduce tal peroxidación y evita el daño progresivo de tejidos, diferenciación celular, inflamación y apoptosis, inclusive si estos son efecto derivado del tratamiento con radio 0 quimioterapia ${ }^{10}$.

c) Paliativos: Su propósito deja de ser curativo, más bien, buscan aliviar otra sintomatología. En este caso, la vitamina $\mathrm{E}$ funciona reduciendo la neurotoxicidad derivada de los tratamientos y evitando sintomatología como fatiga, debilidad y dolor. Por otra parte, otro efecto presentado posterior a los tratamientos es la diarrea, acompañado de sangrado y esteatorrea, para lo cual se sugiere el consumo de probióticos, cuyo fin es la restauración del ecosistema intestinal. Finalmente, hay estudios que sugieren que las selenoproteínas contenidas en el plasma pueden reducir ciertos radicales libres ayudando a la reducción de diarrea mediante la repoblación del intestino ${ }^{10}$.

No obstante, la actividad de dichos suplementos resulta ambigua, puesto que, si bien se han encontrado efectos positivos sobre el desarrollo de cáncer, estos presentan limitaciones en cuanto a las variaciones genéticas y metabólicas, así como el tipo de cáncer sobre el que actúan e inclusive, las concentraciones séricas previas a la neoplasia o al tratamiento de quimio o radioterapia. 


\section{Asociación del consumo de diversos compuestos bioactivos durante el tratamiento de distintos tipos de cáncer}

\section{- Antioxidantes}

El estrés oxidativo es una repercusión de las enfermedades neoplásicas; afectando la función celular de células cancerosas y derivando en angiogénesis tumoral, disminuyendo a su vez antioxidantes endógenos (superóxido dismutasa, glutatión peroxidasa) y exógenos (Vitaminas C, E, $\beta$-carotenos y selenio). Dicho estrés se ve aumentado a lo largo del tratamiento con quimioterapia. En diversos estudios se han encontrado resultados positivos posteriores a la suplementación de antioxidantes, como reducción en la carbonilación proteica y estabilización de los niveles de hemoglobina en pacientes con cáncer cervicouterino ${ }^{4}$.

En 2016, se dirigió un estudio clínico controlado sobre 103 pacientes con cáncer cervicouterino en los estadios B2 y B3, los cuales fueron suplementados con una mezcla de antioxidantes ( $\beta$-caroteno, ácido ascórbico, $\alpha$-tocoferol, levadura de selenio, óxido de zinc, extracto de ginkgo biloba y ginseng) o placebo (azúcar granulada) a lo largo de 4 años, posterior al término del tratamiento antineoplásico; buscando evaluar la disminución de los efectos secundarios propios del tratamiento, así como el riesgo de recurrencia ${ }^{11}$.

Del total de los participantes, únicamente 88 estuvieron disponibles 4 años después, 42 en el grupo de antioxidantes y 46 placebo, sin encontrar diferencias entre los grupos en la recurrencia de cáncer ni metástasis; infiriendo que la suplementación de antioxidantes durante el tratamiento de cáncer no interfiere con la eficacia del mismo. Sin embargo, se encontró una mayor supervivencia en los pacientes suplementados durante el tratamiento oncológico ${ }^{11}$.

Dados los efectos prooxidantes, la suplementación parece no ser recomendable, salvo en casos de deficiencias; mientras que la ingesta diaria recomendada pudiera ser suficiente para obtener resultados, que, al ser contradictorios, requieren una mayor investigación que compruebe tales beneficios.

\section{- Polifenoles}

Son en general, compuestos fitoquímicos con estructuras similares, que se clasifican a su vez en ácidos fenólicos (ácido anacárdico, ácido cafeico, ácido elágico, ácido gálico), con propiedades antiinflamatorias, antioxidantes, anticancerígenas y antimicrobianas; estilbenos (resveratrol y piceatannol) potenciales agentes quimioprotectores, capaces de inhibir iniciación, promoción y progresión tumoral; curcuminoides (curcumina), con efecto antiinflamatorio, antioxidante y anticancerígeno; y flavonoides (Catequinas, antocianinas, isoflavonas) antioxidantes, antiinflamatorias y anticancerígenas ${ }^{12}$ (Figura 1). Su efecto quimiopreventivo y anticancerígeno radica en la reducción del daño celular provocado por estrés oxidativo; sin embargo, estas acciones van a depender de factores como concentración y $\mathrm{pH}^{4}$. En alimentos como la toronja, se han encontrado propiedades antimetastásicas sobre ciertas células ${ }^{13}$.

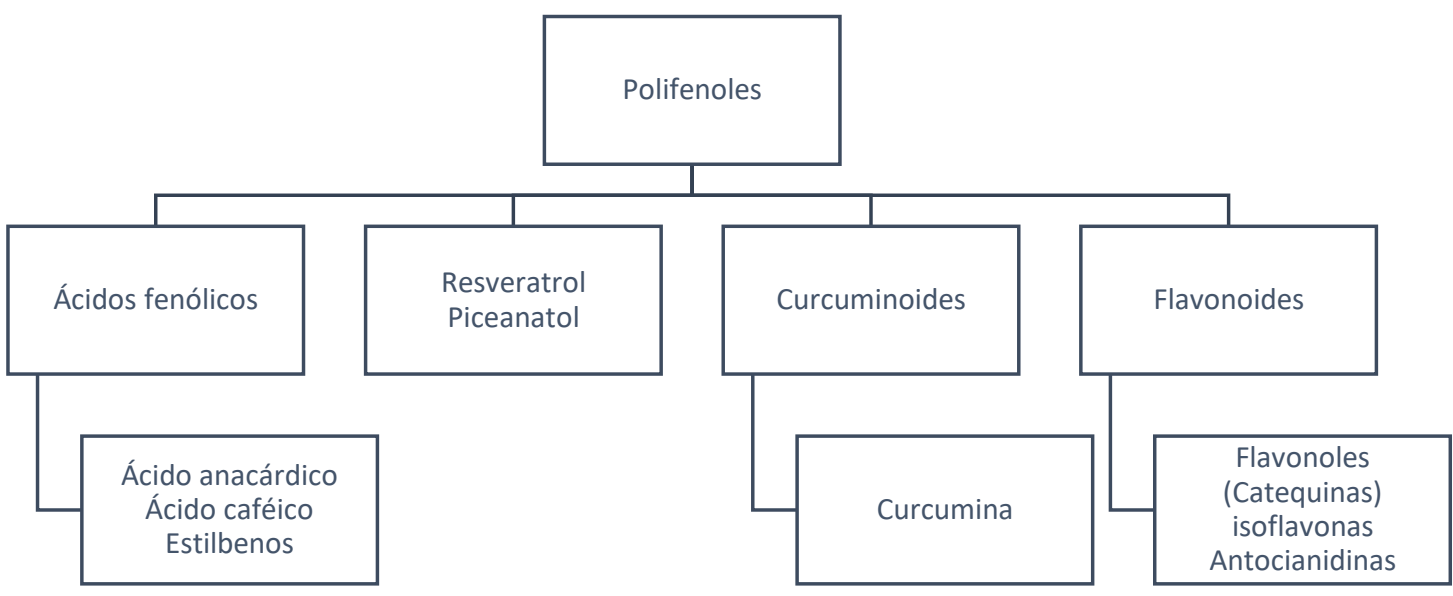

Figura 1 Clasificación de polifenoles. Se muestra la clasificación de los principales polifenoles descritos. Fuente: (Lall et al., 2015)

Entre los ácidos fenólicos con propiedades específicas en el cáncer de próstata se encuentra el ácido anacárdico, inhibidor de angiogénesis tumoral y el ácido cafeico con efecto citotóxico y antiproliferativo ${ }^{12}$.
Los estilbenos, por su parte, como el piceatannol, que se encuentra en algunas moras, cacahuates, vino y piel de uvas, ha mostrado inducir apoptosis además de inhibir la formación y crecimiento tumoral, así como controlar 
actividades celulares involucradas en inflamación y carcinogénesis. El resveratrol ha mostrado capacidad anticancerígena, sin embargo, no se ha determinado una dosis óptima que justifique su uso; por lo que es necesario concretar la evidencia mediante estudios clínicos ${ }^{12}$.

La curcumina; principal curcuminoide, inhibe en el cáncer de próstata la viabilidad celular además de inducir apoptosis y proteger ante invasión y metástasis de células neoplásicas; encontrando una mejor respuesta en análogos de este componente; aunque aún son requeridos mayores estudios ${ }^{12}$.

Los flavonoides comprenden alrededor del $60 \%$ del total de polifenoles dietarios; con alrededor de 4000 variedades 12. Las catequinas se encuentran en el té verde (Camellia sinensis), siendo en mayor proporción (50-75\%) la epigalocatequina-3-galato (EGCG). En el tratamiento de cáncer de mama, se ha demostrado que actúa mediante la disminución de estradiol en sangre, así como antiestrogénico; pudiendo ser útil en el tratamiento de tumores hormono dependientes; así como el aumento de la expresión de genes supresores de tumores p53/p21; además de mantener una acción sinérgica con ciertos medicamentos, aunque, resulta necesario un mayor estudio que consolide dicha evidencia ${ }^{14}$. En cáncer cervicouterino, se ha encontrado efecto promotor de apoptosis y autofagia, además de atenuar los efectos por estrés oxidativo ${ }^{10}$. En cáncer de próstata, inhibe la proliferación tumoral e induce apoptosis ${ }^{12}$. A pesar de que se ha encontrado una relación inversa entre su consumo y la tasa de reaparición tumoral; los estudios resultan ambiguos, puesto que no se han encontrado resultados positivos en cuanto a la suplementación y la evolución tumoral; por lo que, su consumo podría ser únicamente coadyuvante al mantenimiento integral del estado de salud del paciente, dado su efecto en el control de grasa corporal y peso, así como niveles de glucosa y HDL ${ }^{14}$. Los polifenoles presentes en la soja, en forma de isoflavonas; asociadas a la unión de receptores de estrógenos (ER); encontrándose ERa, que estimula la proliferación celular y ER $\beta$ que la inhibe; siendo estos compuestos más afines al segundo tipo. Derivado de su consumo, se ha encontrado un efecto antiangiogénico y antiinflamatorio; además de una sensibilización celular ante terapias de radiación. Por otra parte, se ha relacionado con efectos preventivos de patologías cardiovasculares y obesidad; sin embargo, la elevada ingesta se asocia a la inhibición de topoisomerasa, resultando perjudicial para células normales ${ }^{14}$.

En el caso de las isoflavonas, los resultados son ambiguos; pues los efectos positivos se han encontrado en poblaciones cuyo consumo es mayor y a lo largo de la vida, por otro lado, se han demostrado efectos negativos derivados de un consumo elevado ${ }^{14}$.
Las antocianinas; en alimentos como grosella, uvas, moras y algunas variedades de maíz, con potencial actividad antioxidante, antiinflamatoria y antitumoral; tienen la capacidad de inhibir la proliferación celular ${ }^{13,14}$. Como antioxidantes, se encargan de eliminar los radicales libres reduciendo así, el daño al genoma de células normales por estrés oxidativo y su consiguiente mutación previniendo la aparición de tumores. Por otra parte, las antocianinas pueden controlar la expresión y secreción de factores inflamatorios inhibiendo ciertos factores de transcripción. Algunos estudios han relacionado el consumo de antocianinas con reducción del riesgo de cáncer de colon; aunque, estos resultados han sido desestimados por otros. Esto no quiere decir, que no exista un efecto reductor de daño oxidativo derivado de la ingesta; protegiendo la piel del daño inducido por radioterapia ${ }^{15}$.

- Vitamina E

Naturalmente se encuentra en semillas y frutos secos, así como hierbas de hojas verdes; se han estudiado sus efectos angiogénicos y antitumorales, revelando una disminución de los efectos adversos asociados a tratamientos con radiación. De manera general son conocidas sus propiedades antioxidantes, neuroprotectoras y en el control de los niveles de colesterol; que, aunque no interfieren directamente en el desarrollo tumoral, son útiles en el mantenimiento del estado de salud del paciente ${ }^{14}$. Sin embargo, no hay aún estudios que demuestren posibles efectos negativos en dosis elevadas o mediante suplementación, por lo que no es posible determinar un efecto directo en la evolución y pronóstico de la enfermedad.

\section{- Vitamina D}

La vitamina $D$ o calciferol, se suele encontrar en alimentos como pescados azules, huevo, algunos lácteos y setas. En el tratamiento de cáncer de mama se ha visto que los niveles séricos bajos de esta vitamina estimulan la hormona paratiroidea, derivando en mayores tasas metastásicas óseas; mientras que, altos niveles séricos implicarían menor reaparición tumoral y mayor supervivencia. En este sentido, la disminución en los niveles de hormona paratiroidea se asociaría a fenotipos tumorales menos proliferativos ${ }^{14}$.

Tomando en cuenta el punto anterior, la concentración sérica de vitamina $D$ puede funcionar como un biomarcador que indique la severidad de la enfermedad neoplásica. Sin embargo, dado el riesgo de hipercalcemia se toma el paricalcitol como análogo menos calcémico para suplementación adecuada durante el tratamiento de quimioterapia ${ }^{14}$. Además, la suplementación reporta mejoras en parámetros óseos, que aunque no interfieren directamente en el desarrollo del trastorno neoplásico, 
coadyuvan al mantenimiento del estado de salud general del paciente.

- Fibra ( $\beta$-glucanos)

Parte estructural de la pared celular de avena, cebada, hongos shiitake y maitake, así como levaduras; son polímeros de glucosa con capacidad estimulante inmune, mediante el incremento de tasas de fagocitosis y en el caso de tratamiento de cáncer, capaces de incrementar la eficacia del mismo; además de reducir los procesos de metástasis y apoptosis. Sin embargo, los ensayos existentes que obtienen resultados benéficos son antiguos, por lo que es necesaria investigación actualizada ${ }^{14}$.

El consumo de $\beta$-glucanos es considerado seguro, sin embargo, dada la falta de resultados, no es posible asociarlo a un efecto antineoplásico o coadyuvante; aunado a que, al ser la tasa de absorción intestinal más bien baja, se requeriría de una ingesta elevada.

\section{- $\quad E P A / D H A$}

Ácido eicosapentanoico (EPA) y ácido docosahexaenoico (DHA), son ácidos grasos poliinsaturados de cadena larga omega-3, es decir, su consumo es esencial; y se encuentra en pescados azules y algas. En el caso de cáncer de mama, la ingesta de $\Omega-6$ (aceites vegetales) incrementa el riesgo de padecerlo; por lo que preferir el consumo de $\Omega-3$ podría considerarse un factor de reducción de riesgo ${ }^{14}$.

Durante los tratamientos antineoplásicos, el consumo y suplementación de EPA y DHA es capaz de incrementar la eficacia de compuestos antitumorales, así como la quimiosensibilización de tejidos tumorales, sin afectar las células normales ${ }^{4,14}$, además de controlar apoptosis, oncogénesis, metástasis, y disminuir la respuesta inflamatoria, así como estrés oxidativo y acción citotóxica sobre células cancerosas ${ }^{4}$. Como coadyuvante a los tratamientos antineoplásicos, se ha encontrado una reducción de efectos adversos como anorexia, fatiga, inflamación, neuropatía, resorción ósea y pérdida de masa magra, incrementado la calidad de vida del paciente ${ }^{14,16}$. En el cáncer de páncreas, se ha mostrado un incremento en los efectos antiproliferativos y antinvasivos de ciertos medicamentos ${ }^{16}$.

Existen estudios que sugieren un consumo mínimo de 2 g/día para obtener un beneficio, sin embargo, también se han reportado resultados no óptimos; como incremento de sangrado en niños y adolescentes durante la suplementación de 1-5 g/día de aceite de pescado ${ }^{16}$.

En 2019, un ensayo clínico aleatorizado resaltó los efectos del consumo de ácidos grasos omega-3 durante el tratamiento antineoplásico para pacientes con cáncer, entre los que destacaron la reducción de la sintomatología asociada a la quimioterapia, como fatiga, dolor, alteraciones del sueño, alteraciones gastrointestinales, así como la preservación de masa muscular y el mantenimiento de peso corporal ${ }^{17}$.

En un ensayo que se realizó sobre 53 mujeres con cáncer de mama localizado en un estadio A2-B2, que recibían quimioterapia neoadyuvante; 27 recibieron AGPI $\Omega-3$ (4 capsulas al día en una proporción 2:1 de DHA/EPA, 2.4g), y 26 de ellas pertenecieron al grupo placebo (aceite de semilla de girasol) durante 6 meses de quimioterapia. Al finalizar, no se encontró alguna diferencia significativa en la incidencia de alteraciones hematológicas, sintomatología relacionada a toxicidad gastrointestinal, fatiga, dolor, peso corporal, índice de masa corporal (IMC) ni perfil cardiometabólico. Sin embargo, el grupo suplementado con AGPI $\Omega$-3 mostró una reducción significativa de grasa corporal en comparación con el grupo placebo; además de una mejoría para aquellas que padecían xerostomía. Por lo que se concluyó que la suplementación de AGPI $\Omega-3$ no contribuye a la preservación de la composición corporal, ni a la reducción de efectos derivados de la toxicidad en el tratamiento de cáncer de mama ${ }^{17}$.

Debido a las inconsistencias y ambigüedad de los resultados, se considera necesaria una investigación más amplia, que certifique la utilidad de ácidos grasos como EPA y DHA en tratamientos para el cáncer, ya sea para mantener e incrementar la calidad de vida del paciente, así como por sus potenciales efectos sinérgicos y benéficos.

- Isotiocianatos (ITC)

Son compuestos bioactivos que se encuentran en crucíferas como coles y brócoli; destacando el sulforafano, que mediante regulación de caspasas incrementa la apoptosis de tejidos tumorales. La erucina, con capacidad selectiva sobre células cancerosas; que inhibe y modula enzimas de ciclo celular y procesos angiogénicos, además de inducir apoptosis. Así como algunos indoles (indol-3carbinol) con efecto sinérgico en conjunto con medicamentos para el tratamiento de cáncer de mama, disminuyendo la tasa de crecimiento celular tumoral. También participan en la detoxificación de carcinógenos mediante la inducción de glutatión-s-transferasa 8,13,14, además de suprimir de manera dependiente la adhesión, invasión y migración de células cancerosas mediante regulación de metaloproteinasa ${ }^{13}$. Se ha propuesto como supresor de la proliferación de células tumorales al inhibir la progresión del ciclo celular e inducir apoptosis ${ }^{7}$.

\section{- Lectinas}

Con funciones biológicas diversas, entre las que destacan, antibacteriales y antitumorales. Las lectinas extraídas de ejotes han mostrado una citotoxicidad selectiva. Tal actividad antitumoral se da mediante diversos mecanismos, que incluyen la inducción de apoptosis y necrosis, regulando la producción de iNOS 
(óxido nítrico sintetasa) y liberando citoquinas proinflamatorias; además de contar con propiedades antiproliferativas ${ }^{13}$.

\section{- Probióticos y prebióticos}

Se conoce por probióticos a los organismos vivos que se ingieren en conjunto con algún alimento, siendo su papel obstaculizar la replicación patógena y producción de toxinas; mientras que los prebióticos son productos no digeribles que al ser metabolizados modulan la composición y actividad del microbiota intestinal; cuyo fin es, a su vez, prevenir la invasión del tracto gastrointestinal por bacterias patógenas ${ }^{4}$.

Los tratamientos por radio y quimioterapia pueden conducir, como se ha mencionado antes, a efectos secundarios como diarrea, permeabilidad vascular de la mucosa y alteraciones de la motilidad intestinal 4; que además de restringir la calidad de vida del paciente, puede conllevar a mayores afecciones. Existen estudios que se han encargado de evaluar el efecto de probióticos en la prevención de diarrea inducida por quimioterapia en pacientes con cáncer abdominal o pélvico, encontrando un efecto positivo, además de generar resistencia ante infecciones reduciendo a su vez la necesidad de antibióticos e incidencia de fiebre ${ }^{4}$; resultando de ayuda durante los tratamientos.

\section{- Glutamina}

Se trata de un aminoácido no esencial; en pacientes críticos incrementa la respuesta celular y la función de la mucosa intestinal, mientras que disminuye la tasa de infecciones y duración de estancias hospitalarias. Por otra parte, resulta sumamente importante en el mantenimiento intestinal, siendo precursor de glutatión, clave a su vez, de la cadena antioxidante; además de regular la respuesta inflamatoria mediante el control de producción de citoquinas ${ }^{10}$.

La suplementación de glutamina ha mostrado actividad inflamatoria de tejidos intestinal y en esófago inducidas por radiación, sin embargo, no llega a ser un determinante como protector contra la misma, dado que los resultados clínicos continúan siendo inconsistentes. Por otra parte, se encontró en estudios previos una reducción en el tiempo de duración de diarrea provocada por quimioterapia, aunque no hubo cambios en cuanto a su severidad; además de tener efectos preventivos para neuropatía inducida por quimioterapia. Sin embargo, la glutamina está asociada a efectos negativos. En distintos estudios se han encontrado resultados no favorables; como un incremento en las tasas de recaída tumoral ${ }^{16}$; mientras que, en otro, se hace hincapié en el hecho de que las células cancerosas compiten con el huésped por la glutamina circulante, consumiendo hasta el 50\%, y provocando a su vez una depleción de la misma progresiva al incremento tumoral; siendo indispensable para la proliferación y crecimiento tumoral ${ }^{4}$. Por lo que, siendo los resultados tan ambiguos, se requiere un mayor control de eficacia y seguridad para su recomendación.

En la tabla 2, se resumen los efectos de los compuestos bioactivos durante tratamientos neoplásicos sobre distintos tipos de cáncer.

Tabla 2. Compuestos bioactivos y su efecto sobre tratamientos antineoplásicos

\begin{tabular}{|c|c|c|}
\hline Sustancia & Efecto demostrado sobre distintos tipos de cáncer & Referencias \\
\hline Antioxidantes & $\begin{array}{l}\text { General } \\
\text { - } \quad \text { Actúa contra el estrés oxidativo } \\
\text { - } \quad \text { Reducción de carbonilación proteica }\end{array}$ & 4,11 \\
\hline $\begin{array}{l}\text { Polifenoles (Ácidos } \\
\text { fenólicos, estilbenos, } \\
\text { curcuminoides, } \\
\text { flavonoides) }\end{array}$ & $\begin{array}{l}\text { General } \\
\text { - } \quad \text { Prevención de carcinogénesis en células normales } \\
\text { - Reducción del daño al genoma debido a estrés oxidativo, } \\
\text { - } \text { previniendo la aparición de tumores. } \\
\text { - } \quad \text { Inhibición de la mutación de células normales; y proliferación } \\
\text { - } \quad \text { selectiva de células cancerosas. } \\
\text { Cáncer de mama } \\
\text { - Modulación de rutas de señalización y ciclo celular } \\
\text { - Efecto angiogénico, antiinflamatorio, y sensibilización ante } \\
\text { - } \quad \text { radiación. } \\
\text { - Aumenta la expresión de genes supresores de tumores } \\
\text { - Inhibición de proliferación celular } \\
\text { - Supresión de estrés oxidativo e inflamación }\end{array}$ & $\begin{array}{l}4, \quad 12,13, \\
14,15\end{array}$ \\
\hline
\end{tabular}


Vitamina $\mathrm{E}$

Vitamina D

Fibra

( $\beta$-glucanos)

EPA / DHA

Isotiocianatos

Lectinas
Cáncer de hígado

- Propiedad antimetastásica

- Actividad inhibidora de tumores en una etapa temprana de carcinogénesis

- Inducción de apoptosis

Cáncer de próstata

- Inhibe angiogénesis tumoral.

- Antiinflamatorio, antioxidante

- Inhibe eventos celulares asociados a carcinogénesis, incluyendo iniciación y progresión tumoral

- Regulación de metástasis, apoptosis y angiogénesis

- Modulación de respuesta inflamatoria y proliferación celular

Cáncer de mama

- Reducción de fibrosis del tejido irradiado

- Restauración de actividad antioxidante

Cáncer de mama

- Baja concentración produce mayores tasas metastásicas ósea

- Altos niveles derivan en una menor reaparición tumoral

- Disminución de niveles de hormona paratiroidea, asociada a fenotipos tumorales menos proliferativos

Cáncer de aparato digestivo

- Efecto inmunomodulador

- Incremento de eficacia en terapias biológicas

- Aumento de tasas de fagocitosis, potenciación de actividad de anticuerpos monoclonales

- Reducción de procesos metastásicos y apoptóticos.

General

$4,14,16,17$

- Toxicidad selectiva a células cancerosas.

- Disminución de oxidación sistémica y estrés oxidativo.

- Actividad antineoplásica mediante inducción de apoptosis.

Cáncer de mama

- Quimiosensibilización de tejidos tumorales frente a agentes anticancerígenos

- Cambios en la expresión génica asociada a procesos metastásicos.

Cáncer de colon

- Propiedad anticaquéctica

General

- Inducción de apoptosis sobre células tumorales.

Cáncer de mama

- Aumento de la apoptosis en tejidos tumorales

- Promoción de procesos antiproliferativos en células cancerosas, pero no en células no transformadas

- Potencial antiinflamatorio, antiangiogénico y proapoptótico

Cáncer de hígado

- Detoxificación de compuestos carcinógenos

- Supresión de invasión, adhesión y migración celular.

General

- Actividad antitumoral y antiproliferativa.

- Inducción de apoptosis, autofagia y necrosis

- Evita la producción y la liberación de citoquinas proinflamatorias. 


\begin{tabular}{|c|c|c|}
\hline $\begin{array}{l}\text { Probióticos } \\
\text { prebióticos }\end{array}$ & $y$ & $\begin{array}{l}\text { General } \\
\text { - Inhibe replicación de patógenos y producción de toxinas } \\
\text { - Protección ante diarrea inducida por radiación }\end{array}$ \\
\hline Glutamina & & $\begin{array}{l}\text { General } \\
\text { - Regulación de respuesta inflamatoria } \\
\text { - Reducción en afecciones secundarias, como diarrea }\end{array}$ \\
\hline
\end{tabular}

A lo largo de esta revisión se encontraron publicaciones que si bien, intentaron comprobar los efectos benéficos del consumo de ciertos nutrientes, obtuvieron resultados poco prometedores. Tal es el caso del Ganoderma lucidum (Reishi). Se trata de un hongo de origen asiático con propiedades de soporte inmune. En 2016, un estudio buscó evaluar los efectos de respuesta tumoral, inmune y calidad de vida de pacientes con cáncer ante el consumo de G. Iucidum. Encontrando a través de un metaanálisis que aquellos que lo habían consumido a la par del tratamiento con quimio/radioterapia tuvieron una mejor respuesta en comparación con aquellos que tuvieron el tratamiento solo. Sin embargo, el consumo en sí mismo del hongo no demostró algún efecto, por lo tanto, la evidencia obtenida no justifica el uso del hongo reishi como coadyuvante en el tratamiento de cáncer ${ }^{18}$.

\section{EPIGENÉTICA Y CÁNCER}

El cáncer es producto de una modificación a nivel celular, que deriva en una alteración funcional y morfológica de las mismas; por su parte, se sabe que la nutrición se encuentra ligada a la regulación y proliferación celular y hormonal, así como procesos de diferenciación, inflamación e inclusive, inmunidad y apoptosis. La epigenética, interviene entonces, en la modulación de genes a partir de los factores de exposición en el medio, entre los cuales se encuentran los dietéticos ${ }^{19}$. De esta manera, se han estudiado sustancias con efecto epigenético que intervienen en el desarrollo o prevención de cáncer, que se muestran en la tabla 3.

Tabla 3. Interacciones entre compuestos bioactivos y epigenética en el desarrollo de cáncer

\begin{tabular}{|c|c|c|}
\hline Sustancia & Interacción epigenética & Referencias \\
\hline Folatos & $\begin{array}{l}\text { Metilación de ADN. El consumo de alcohol aumenta el riesgo de la metilación de } \\
\text { genes supresores de tumores. } \\
\text { En mujeres pre menopáusicas, el consumo sugiere un menor riesgo de cáncer de } \\
\text { mama. }\end{array}$ & 19 \\
\hline Polifenoles & $\begin{array}{l}\text { Hipometilación o supresión de la metilación en genes supresores de tumores, así } \\
\text { como antioxidantes. } \\
\text { Inhibición de la invasión tumoral y la angiogénesis en modelos animales. }\end{array}$ & 19 \\
\hline Selenio & $\begin{array}{l}\text { Remodelación de la cromatina. Reactivación de genes con capacidad de modificar } \\
\text { la acetilación y desacetilacion de histonas. }\end{array}$ & 7 \\
\hline Isotiocianatos & Inhibición del regulador transcripcional de la respuesta inflamatoria sistémica. & 20 \\
\hline Vitamina D & $\begin{array}{l}\text { Regulación de metilación y modulación de expresión génica. Reexpresión de genes } \\
\text { diana antiproliferativos. }\end{array}$ & 21 \\
\hline
\end{tabular}

\section{Conclusión}

El incremento en la incidencia de enfermedades neoplásicas y las eventuales consecuencias de su tratamiento, han orillado a la búsqueda de alternativas que mantengan la calidad de vida de los pacientes. Se ha encontrado en la alimentación un factor determinante antes, durante y después de su aparición y procedimientos; por lo que la calidad de la misma es cada vez más, un objeto de estudio.

En la presente revisión se han expuesto los efectos de específicos compuestos bioactivos, los cuales se ha recomendado como adyuvantes en el tratamiento del cáncer. No obstante; muchos de los artículos evaluados durante esa revisión muestran, si bien, resultados positivos; ciertas ambigüedades e inclusive resultados que no indican algún beneficio en el consumo ni suplementación de compuestos bioactivos. Lo cual podría deberse a la genética de cada individuo.

Por lo que en conclusión se recomienda el uso supervisado y racionalizado de antioxidantes, polifenoles, fibra y ácidos grasos $\Omega-3$, que ayuden a sobre llevar los 
efectos de los diversos tratamientos. Sin embargo, es necesario una investigación más amplia y con un mayor control que permita establecer tanto la existencia o no, de beneficios como la dosis recomendada y la temporalidad en la que resultaría más útil su consumo.

\section{Referencias}

[1] Aldaco-Sarvide F, Pérez-Pérez P, Cervantes-Sánchez G, TorrecillasTorres L, Erazo-Valle-Solis A, Cabrera-Galeana P, Motola-Kuba D, Anaya P, Rivera-Rivera S, Cárdenas-Cárdenas E. Mortalidad por cáncer en México: Actualización 2015. Gac. Mex. Oncol. 2018; (17): 28-34.

[2] Salamanca-Fernández E, Rodríguez-Barranco M, Sánchez M. La dieta como causa del cáncer: Principales aportaciones científicas del Estudio Prospectivo Europeo sobre Nutrición y Cáncer (EPIC). Nutr. Clin. Med. 2018; XII (2):61-79.

[3] Villegas C, García M. La nutrición en la inmunidad y el cáncer. Rev. Argent. Endocrinol. Metab. 2013; 51(1): 30-36.

[4] Serna-Thomé G, Castro-Eguiluz D, Fuchs-Tarlovsky V, Sánchez-López M, Delgado-Olivares L, Coronel-Martínez J, Molina-Trinidad E, De la Torre M, Cetina-Pérez L. Use of functional foods and oral supplements as adjuvants in cancer treatment. Rev. Inves. Clin. 2018; 70(3): 136146.

5[4] Martínez-Rodríguez T, González W, Atencio J, Padilla I, Machacon S Caracterización del uso de terapias complementarias y alternativas relacionadas con la alimentación en pacientes oncológicos de un centro hospitalario de Cartagena, Colombia. Nutr. Clín. Hosp. 2020; 40(2): 149-158.

[6] Muniraj N, Siddharth S, Sharma D. Bioactive Compounds: MultiTargeting Silver Bullets for Preventing and Treating Breast Cancer. Cancers. 2019; 11(10): 1563 .

[7] Campos-Vega R, Oomah BD, Loarca-Piña G, Vergara-Castañeda HA. Common Beans and Their Non-Digestible Fraction: Cancer Inhibitory Activity-An Overview. Foods. 2013; 2(3):374-392.

[8] Martínez-López E, García-García M, Campos-Pérez W, GonzálezBecerra K. Genómica nutricional: Conceptos y expectativas. Rev. Endocr. Nutr. 2013; 21(1): 22-34.

[9] Molina R. El paciente oncológico del siglo XXI. Maridaje terapéutico Nutrición-Oncología. Nutr. Hosp. 2016; 33(1): 3-10.

[10] Arellano A, Jiménez F, Salcedo V. Suplementos dietéticos como tratamiento en el cáncer cervicouterino: Revisión sistemática. Nutr. Hosp. 2013; 28(6): 1770-1780

[11] Álvarez-Altamirano K, Mendoza-Hernández A, Carcoba-Tenorio C, García-García J, Fuchs-Tarlovsky V. Antioxidant supplementation during oncology treatment has no effect on cervical cancer recurrence. Nutr. Hosp. 2016; 33(2): 411-414.

13[12] Lall R, Syed D, Adhami V, Khan M, Mukhtar H. Dietary Polyphenols in Prevention and Treatment of Prostate Cancer. Int. J. Mol. Sci. 2015; 16(2): 3350-3376.

14[13] Zhou Y, Li Y, Zhou T, Zheng J, Li S, Li H. Dietary Natural Products for Prevention and treatment of liver cancer. Nutrients. 2016; 8(3): 156.

15[14] Dahdouh S, Bermejo L, López B, Palma S, Peregrina B, Santamaría B, Gómez C. Revisión de la evidencia científica sobre el papel de compuestos bioactivos de alimentos como coadyuvantes a los tratamientos antineoplásicos de cáncer de mama. Rev. Esp. Nutr. Comunitaria 2017; 23(2).

16[15] Bo-Wen L, Cheng-Chen G, Hai-Fei S, Ying-Yu C. Effects of anthocyanins on the prevention and treatment of cancer. Br. J. Pharmacol. 2017; 174(11): 1226-1243.

17[16] Arends J, Bachmann P, Baracos V, Barthelemy M, Hartmut B, Bozzeti F, Fearon K, Hütterer E, Isenring E, Kaasa S, Krznaric Z, Laird
B, Larsson M, Laviano A, Mühlebach S, Muscaritoli M, Oldervoll L, Ravasco P, Solheim T, Strasser F, de van der Schueren M, Preiser, J. ESPEN guidelines on nutrition in cancer patients. Clin. Nutr. 2017; 36(1), 11-48.

18[17] De la Rosa O, Meneses A, Ruiz H, Astudillo H, Bargalló E, LaraMedina F, Alvarado A, Matus-Santos J, Flores-Díaz D, Oñate-Acuña L, Gutiérrez-Salmeán G, Ruíz E, Ibarra, A. Effects of omega-3 fatty acids supplementation on neoadjuvant chemotherapy-induced toxicity in patients with locally advanced breast cancer: a randomized, controlled, double-blinded clinical trial. Nutr. Hosp. 2019; 36(4): 769766.

19[18] Jin X, Ruiz J, Man-Yeun D, Chan G. Ganoderma lucidum (Reishi mushroom) for cancer treatment. Cochrane database of Syst. Rev. 2012; 13(6):CD007731.

20[19] Hernando-Requejo O, García del Quinto H, Rubio M. Nutrición como factor epigenético regulador del cáncer. Nutr. Hosp. 2019; 36(Extr.3): 53-57.

21[20] Caballero-Gutiérrez L, Gonzáles G. Alimentos con efecto antiinflamatorio. Acta Méd. Peruana 2016; 33(1), 50-64.

22[21] Fetahu I, Höbaus J, Kállay E. Vitamin D and the epigenome. Front Physiol. 2014; 5: 164 